\title{
A iniciativa digital CONVIDE-i9 no combate à infodemia de COVID-19: breves apontamentos de atuação
}

\section{The digital initiative CONVIDE-i9 in the fight against COVID-19 infodemia: brief action points}

\author{
Fernanda Percia França ${ }^{1}$, Denise Oliveira de Araújo² ${ }^{2}$, Márcio Bezerra da Silva ${ }^{3}$ \\ ${ }^{1}$ Universidade de Brasília - UnB, Brasília, DF, Brasil. ORCID: http://orcid.org/0000-0002-9844-769X \\ 2 Universidade de Brasília - UnB, Brasília, DF, Brasil. ORCID: https://orcid.org/0000-0003-4033-7882 \\ ${ }^{3}$ Universidade de Brasília - UnB, Brasília, DF, Brasil. ORCID: https://orcid.org/0000-0002-0052-7174
}

Autor para correspondência/Mail to: Fernanda Percia França, fernanda10nov@hotmail.com

Recebido/Submitted: 11 de setembro de 2020; Aceito/Approved: 18 de novembro de 2020

Copyright (c) 2020 França, Araújo \& Silva. Todo o conteúdo da Revista (incluindo-se instruções, política editorial e modelos) está sob uma licença Creative Commons Atribuição-NãoComercial-Compartilhalgual 3.0 Não Adaptada. Ao serem publicados por esta Revista, os artigos são de livre uso em ambientes educacionais, de pesquisa e não comerciais, com atribuição de autoria obrigatória. Mais informações em http://revistas.ufpr.br/atoz/about/submissions\#tcopyrightNotice.

\begin{abstract}
Resumo
Introdução: Enseja apresentar apontamentos resultantes de uma experiência acerca da catalogação de materiais na biblioteca virtual CONVIDE-i9, um produto informacional confiável no combate à desinformação, fake news e infodemia. Visa pontuar características da biblioteca; elencar as temáticas cobertas; quantificar as publicações cadastradas por categoria e relatar a atuação pessoal no cadastro de materiais. Método: Caracteriza-se como uma pesquisa descritiva e bibliográfica, com abordagem quanti-qualitativa de coleta de dados na biblioteca virtual CONVIDE-i9, no período de maio até junho de 2020. Resultados: Apresenta a CONVIDE-i9 como uma colaboração entre instituições de informação nacionais e bibliotecários voluntários, cujo fito é ser uma fonte de informações confiáveis no âmbito da COVID-19 e temáticas relacionadas; cobre oito áreas temáticas, como "apoio ao teletrabalho", "fake news e phishing" e "protocolos e recomendações", possui 294 materiais cadastrados; elenca que a atuação pessoal culminou no cadastro de 102 itens; resulta na voluntária que possui o maior número de registros cadastrados até 01/07/2020; e relata afinidades temáticas cunhadas a partir da experiência na biblioteca, sobre saúde mental, transtornos alimentares, orientações para bibliotecas públicas diante da COVID-19 etc. Conclusão: Evoca, portanto, a importância dos bibliotecários na disponibilização de um ambiente digital que alcance o maior número de pessoas geograficamente dispersas, especialmente em um cenário de pandemia, promovendo o bem-estar e a disseminação de informações averiguadas e posteriormente publicitadas sobre a COVID-19 em variadas perspectivas.
\end{abstract}

Palavras-chave: Bibliotecário; Biblioteca virtual; Desinformação; Infodemia; Pandemia; COVID-19

\begin{abstract}
Introduction: Presents notes resulting from an experience about the cataloging of materials in the virtual library CONVIDE-i9, a reliable informational product in the fight against misinformation, fake news and infodemia. Points out characteristics of the library; list the topics covered; quantify the publications registered by category and report the personal performance in the material register. Method: It is characterized as descriptive and bibliographic research, with a quantitative and qualitative approach to data collection in the virtual library CONVIDE-i9, from May to June 2020. Results: Presents CONVIDE-i9 as a collaboration between national information institutions and volunteer librarians, whose aim is to be a source of reliable information within the scope of COVID-19 and related topics; covers eight thematic areas, such as "support for teleworking", "fake news and phishing" and "protocols and recommendations"; it has 294 registered materials; lists that personal performance culminated in the registration of 102 items; results in the volunteer who has the largest number of records registered until 01/07/2020; and reports thematic affinities coined from experience in the library, on mental health, eating disorders, guidelines for public libraries before COVID-19, etc. Conclusions: Therefore, it mentions the importance of librarians in providing a digital environment that reaches the largest number of people geographically dispersed, especially in a pandemic scenario, promoting well-being and the dissemination of investigated and subsequently publicized information about COVID-19 in varied perspectives.
\end{abstract}

Keywords: Librarian; Virtual Library; Disinformation; Infodemia; Pandemic; COVID-19

\section{INTRODUÇÃO}

A emergência da Sociedade da Informação, no final do século XX, marca-se por transmutações econômicas, organizacionais e técnicas, cujos reflexos, em países industrializados, atingem desde o eixo político-social até o científico-acadêmico, nesse caso, com cerne nos avanços tecnológicos em áreas como as telecomunicações (Werthein, 2000). Entre as características do supracitado marco está o papel assumido pela informação na sociedade, que passa a ser vista como matéria-prima, inclusive para manipulações, em consequência da grande penetrabilidade, flexibilidade e convergência das tecnologias (Werthein, 2000).

A manipulação de informações pode ser identificada em um conjunto de produtos e serviços, que não só facilitam as formas de interação indivíduo-indivíduo e indivíduo-meio, como redefinem comportamentos, fomentados pela facilitação de práticas como a adoção do comércio eletrônico, do Internet Banking, das chamadas de vídeo, do vídeo-on-demand (popularizado pelos serviços de streaming) das bibliotecas digitais ${ }^{1}$ etc. Esta suposta facilitação,

\footnotetext{
${ }^{1}$ Sistema de informação que "[...] armazena documentos e informações em forma digital em sistema automatizado, [...] em rede, que pode ser consultado [remotamente]" (Cunha \& Cavalcanti, 2008)
} 
adjunta à manipulação, tem o potencial tanto de aprofundar as desigualdades sociais (Werthein, 2000) quanto incorrer na gênese do que se chama desinformação.

A desinformação está intimamente relacionada ao que se conhece como fake news ${ }^{2}$ (notícias falsas, em tradução livre), advindas da disseminação de informações imprecisas ou carentes de autenticidade, visando, deliberadamente, ludibriar quem entra em contato com essas (Organização Pan-Americana da Saúde, 2020, Werthein, 2000). O ambiente on-line propicia um terreno fértil para a desinformação, sendo websites e redes sociais uma espécie de epicentro da questão. Existe um significativo consumo de notícias aliadas a eventos de grande comoção, como eleições, impulsionadores às fake news e incidindo na formação da opinião pública (Delmazo \& Valente, 2018). Desta forma, um exemplo prático da tríade citada (mídias digitais, fake news e evento de grande comoção) se materializa com o surto da COVID- $19^{3}$.

No fim de 2019, intensificando-se no decorrer de 2020, o mundo foi surpreendido pelo surto da COVID-19, que culminou em uma pandemia. Neste cenário, além dos cuidados necessários para evitar o contágio da doença, a humanidade passa a se preocupar com a propagação de fake news, qualificando o conceito de infodemia, ou seja, o aumento expressivo, em um curto espaço de tempo, de informações relacionadas a um determinado tópico, neste caso, a COVID-19 (Organização Pan-Americana da Saúde, 2020), sendo muitas apresentadas de maneira inverídica.

Torna-se, portanto, imperativa a manifestação de agentes capazes de mitigar os efeitos da desinformação e infodemia que, dentre outros fatores, podem incidir diretamente sob a saúde mental da população, bem como potencializar os riscos de contaminação pela COVID-19 (Organização Pan-Americana da Saúde, 2020). O cenário evoca profissionais da informação como os bibliotecários, enquanto indivíduos capacitados a lidar com documentos e informações de naturezas distintas, realizar o tratamento temático, classificatório e descritivo dessas e subsidiar no suprimento de lacunas informacionais de usuários, que variam de infantes a pesquisadores/cientistas. Pensar no bibliotecário em tempos de tecnologias da comunicação e informação (TIC) o rotularia como um cybrarian (Cunha \& Cavalcanti, 2008), vigilante a autenticidade e disseminação de informações.

Defronte às possíveis consequências da desinformação e da infodemia, e consciente do papel do bibliotecário frente ao atual cenário de pandemia, a Associação dos Bibliotecários e Profissionais da Ciência da Informação do Distrito Federal (ABDF), em parceria com a Federação Brasileira de Associação de Bibliotecários (FEBAB) e bibliotecários voluntários, desenvolveu e promoveu a inserção de materiais na biblioteca virtual intitulada CONVIDE-i9. A biblioteca virtual foi criada para promover "[...] a gestão da informação para apoiar a sociedade brasileira e internacional. Nosso maior objetivo é ser uma fonte de informações seguras e confiáveis" (ABDF, 2020a).

O breve relato de experiência objetivou apresentar impressões sobre o trabalho realizado na catalogação de materiais na biblioteca virtual CONVIDE-i9, como um produto de informação confiável sobre a COVID-19 e temáticas relacionadas. Precisamente, objetivou-se pontuar características da biblioteca, elencar as temáticas cobertas, quantificar as publicações cadastradas por categoria e relatar a atuação pessoal no cadastro de materiais na biblioteca.

\section{PROCEDIMENTOS METODOLÓGICOS E RESULTADOS}

Do ponto de vista dos objetivos, a pesquisa se caracteriza como descritiva; enquanto na ótica dos procedimentos técnicos, o estudo se apresenta como bibliográfico, adotando as abordagens de coleta de dados quanti-qualitativa na biblioteca virtual CONVIDE-i9, incluindo um corpus de 102 itens cadastrados no período de atuação como voluntária, correspondente ao espaço temporal de maio até junho de 2020.

Focada na pandemia atual, a CONVIDE-i9 constitui-se por materiais diversos, como notícias, mapas, eventos (gratuitos), protocolos, artigos, fake news, recomendações, painéis nacionais e internacionais etc., organizados em oito categorias, sendo: "apoio ao teletrabalho", "pausa para o café", "fake news e phishing", "pesquisas brasileiras", "legislação", "protocolos e recomendações", "para as crianças" e "vamos aprender algo?". Os materiais divulgados intencionam não apenas informar/atualizar sobre conteúdos relevantes à COVID-19, mas divulgar atividades (diferentes) que podem ser realizadas durante a pandemia.

$\mathrm{Na}$ interface inicial, as publicações cadastradas podem ser filtradas por palavras-chave, buscadas por termos, acessadas via categorias e identificadas entre os itens mais recentes. Ainda na interface inicial é possível identificar os colaboradores mais ativos e os termos mais utilizados em buscas na plataforma.

Entre as oito categorias, a biblioteca virtual possui 294 materiais cadastrados, tendo a seção "Vamos aprender algo?" contendo o maior quantitativo, ou seja, 130 materiais (ABDF, 2020a). As categorias, suas ementas e correspondentes quantidades de materiais cadastrados são apresentados no Quadro 1.

\footnotetext{
${ }^{2}$ Fenômeno caracterizado por alguns como um tipo de desinformação política, que é marcado pela veiculação intencional, geralmente por figuras influentes, de notícias que podem ser comprovadamente identificadas como falsas e que normalmente visam reforçar uma visão de mundo (Delmazo \& Valente, 2018).

${ }^{3}$ Doença causada pelo vírus SARS-CoV-2
} 


\begin{tabular}{|c|l|c|c|}
\hline Categorias & \multicolumn{1}{|c|}{ Ementas } & $\begin{array}{c}\text { Quantidade de } \\
\text { publicações } \\
\text { cadastradas }\end{array}$ & $\begin{array}{c}\text { Atuação no } \\
\text { cadastro de } \\
\text { publicações }\end{array}$ \\
\hline $\begin{array}{c}\text { Apoio ao } \\
\text { teletrabalho }\end{array}$ & Práticas e questões focadas no apoio ao trabalho à distância. & 8 & - \\
\hline $\begin{array}{c}\text { Pausa para } \\
\text { o café }\end{array}$ & $\begin{array}{l}\text { Dicas de organização (para leitura e ambiente de trabalho), } \\
\text { aplicativos, festivais, canais do Youtube, redes sociais, } \\
\text { podcasts e catálogos disponíveis. }\end{array}$ & 36 & 14 \\
\hline $\begin{array}{c}\text { Fake News } \\
\text { Phishing }\end{array}$ & $\begin{array}{l}\text { Notícias falsas, dicas de segurança e golpes/ataques via } \\
\text { e-mail e internet. }\end{array}$ & 20 & 2 \\
\hline $\begin{array}{c}\text { Pesquisas } \\
\text { brasileiras }\end{array}$ & $\begin{array}{l}\text { Investigações/materiais realizados por pesquisadores } \\
\text { brasileiros e internacionais sobre a COVID-19. }\end{array}$ & 29 & - \\
\hline $\begin{array}{c}\text { Legislação } \\
\text { Legislações, decretos, portarias e resoluções em torno da } \\
\text { COVID-19. }\end{array}$ & 14 & 2 \\
\hline $\begin{array}{c}\text { Protocolos e } \\
\text { recomendações }\end{array}$ & $\begin{array}{l}\text { Recomendações webinars, protocolos e diretrizes de } \\
\text { diversas federações e associações. }\end{array}$ & 43 & 2 \\
\hline Para as crianças & $\begin{array}{l}\text { Atividades, projetos, livros e contações de história } \\
\text { disponíveis para crianças durante a pandemia. }\end{array}$ & 14 & 74 \\
\hline $\begin{array}{c}\text { Vamos } \\
\text { aprender algo? }\end{array}$ & $\begin{array}{l}\text { Campanhas, cursos, acervos, associações, centros, visitas } \\
\text { virtuais e informações que estão sendo divulgados durante a } \\
\text { pandemia em diversos segmentos sociais. }\end{array}$ & 130 & 74 \\
\hline
\end{tabular}

Quadro 1. Categorias da biblioteca virtual CONVIDE-i9

Fonte: Dados da pesquisa, 2020

Para atuar na biblioteca virtual, a ABDF produziu e disponibilizou um documento on-line com os materiais a serem cadastrados, organizados por categorias. O cadastro se deu conforme a sequência dos materiais no documento norteador, preenchendo campos como descrição, título, data de publicação web link, origem da informação (fonte), palavras-chave ${ }^{4}$ e arquivo relacionado. Destaca-se que, no cadastro, alguns materiais receberam uma espécie de destaque, a partir da informação "recomendado pela FEBAB".

Quanto a motivação em contribuir com o projeto, primeiro, deu-se pela iniciativa de informar, de forma confiável e segura, conteúdos relacionados à COVID-19, especialmente no aspecto das fake news. Em segundo, a proposta engloba temas que são interesses pessoais de pesquisa que vêm sendo estudados, como ambientes web e repositórios digitais, especialmente os institucionais e de dados. Sendo assim, identificou-se um local para colocar em prática os estudos, na reunião de informações fidedignas, passando, em seguida, por uma verificação da ABDF, para garantir a confiabilidade das informações cadastradas.

Referente à atuação pessoal na CONVIDE-i9, foram cadastrados 102 itens (Quadro 1), de diversas temáticas, tornando-se, até o dia 01/07/2020, a profissional de maior contribuição na equipe. Entre os materiais cadastrados fomentou-se um maior interesse por informações sobre saúde mental, devido ao atual momento de isolamento social, a partir do projeto "Proteja sua saúde mental em tempos de coronavírus", do Instituto Vita Alere, com apoio das redes sociais Facebook e Instagram. De acordo com a ABDF (2020b), o Instituto, em suas contas nas mencionadas redes sociais e em seu website, compartilhará dicas sobre saúde mental.

Diante do isolamento social, criou-se interesse por programas sobre distúrbios, como o intitulado "AMBULIM Programa de Transtornos Alimentares do HCFMUSP", do Instituto de Psiquiatria do Hospital das Clínicas da Faculdade de Medicina da Universidade de São Paulo (IPq/HCFMUSP). Conforme a ABDF (2020c), o projeto foca em transtorno alimentares e atua em questões referentes a moradia e medicamentos de seus pacientes.

Também se criou o interesse, diante da realização de estudos recentes, por recomendações técnicas para o funcionamento de bibliotecas públicas no país, como as promovidas pela Coordenação-Geral do Sistema Nacional de Bibliotecas Públicas (SNBP) (ABDF, 2020d) e pela Associação Brasileira de Normas Técnicas (ABNT) (ABDF, 2020e). Segundo a ABDF, as recomendações da Coordenação-Geral do SNBP focam no combate à disseminação e contaminação provocadas pela COVID-19, enquanto a ABNT disponibilizou 32 normas gratuitas para contribuir no combate ao novo coronavírus.

Legislações relativas à COVID-19 também se tornaram interesse pessoal a cada material cadastrado na biblioteca virtual, na intenção de acompanhar e compreender como os governos do país estão lhe dando com a pandemia. Neste sentido, a ABDF menciona a página da Procuradoria-Geral do Estado do Rio de Janeiro (PGERJ), a qual reúne legislações associadas ao novo coronavírus, entre federais, estaduais e municipais.

\footnotetext{
${ }^{4}$ Campo de seleção (lista de palavras-chave previamente cadastradas). Necessitando da inclusão de palavras-chave não presentes na lista, acessa-se o campo "sugerir nova palavra-chave", separada por vírgula entre as expressões. Cada material recebeu de três a cinco palavras-chave, mas não existe quantidade estipulada.
} 
O interesse por informações clínicas sobre a COVID-19, que já existia, foi se fortalecendo durante o acesso a conteúdos e fontes sobre a temática na alimentação da CONVIDE-i9. Baseado na ABDF (2020f), menciona-se a National Library of Medicine (2020) que, em seu website, disponibiliza dados, notícias, estudos clínicos etc. sobre o novo coronavírus.

Por fim, diante da iniciativa de empresas nacionais e internacionais surgiu o interesse por informações referentes à amenização de regras de direitos autorais em tempos de pandemia. Sendo assim, a ABDF (2020g) cita a "Oxford University Press - COVID-19", ao flexibilizar suas regras de direitos autorais, beneficiando pesquisadores, profissionais da área médica, formuladores de políticas entre outros que estão trabalhando no enfrentamento à pandemia.

\section{CONCLUSÕES}

Durante o período de atuação voluntária na biblioteca virtual, foi possível absorver diferentes informações sobre o novo coronavírus, assim como os seus impactos no mundo. Neste contexto, destaca-se, como principal conhecimento adquirido, as formas de prevenção e iniciativas geradas pelo mundo no combate à pandemia, como as diversas maneiras adotadas por empresas e pessoas, como elas estão lidando com a COVID-19, ponto essencial para os profissionais da informação, como os bibliotecários, na disseminação de conteúdos na comunidade que lhe é assistida, diante da imensidão de informações, entre verdadeiras e fake news, publicados nos mais diversos canais digitais.

As dicas (práticas) de como trabalhar remotamente, o foco das empresas na segurança de seus colaboradores e clientes, o modo como as informações estão sendo disseminadas e de que forma as instituições, como as bibliotecas e museus, estão se conectando com os seus usuários, ofertando espaços virtuais para que haja uma interação, se apresentam como uma maneira de "ocupar" o dia das pessoas na quarentena.

A CONVIDE-i9 continua ativa, contando com 12 colaboradores, todos bibliotecários. Ao longo da pandemia, mudanças no foco dos conteúdos foram ocorrendo. Enquanto no início o conteúdo tratava da mitigação da desinformação (fake news), posteriormente passaram a tratar da retomada gradual das atividades, como as econômicas e comerciais. Os apontamentos relatados, de maneira sintética, representam a experiência vivida na CONVIDE-i9, a qual ofertou, na prática, subsídios informacionais para o retorno seguro ao trabalho presencial e em como se prevenir tanto em casa quanto no ambiente profissional. Evoca, portanto, a importância dos bibliotecários na disponibilização de um ambiente digital que alcance o maior número de pessoas geograficamente dispersas, especialmente em um cenário de pandemia, promovendo o bem-estar e a disseminação de informações averiguadas e posteriormente publicitadas sobre a COVID-19 em variadas perspectivas. 


\section{REFERÊNCIAS}

ABDF. (2020a). Biblioteca digital convide-i9. Recuperado de http://abdf.org.br/biblioteca-virtual/biblioteca-virtual -convide-i9

ABDF. (2020b). Proteja sua saúde mental em tempos de coronavírus. Recuperado de http://abdf.org.br/biblioteca -virtual/biblioteca-virtual-convide-i9/vamos-aprender -algo/proteja-sua-saude-mental-em-tempos-de-coronavirus

ABDF. (2020c). Ambulim - programa de
transtornos alimentares do hcfmusp.
rado de hecupe-
biblioteca-virtual-convide-i9/vamos-aprender-algo/
ambulim-programa-de-transtornos-alimentares-do-hcfmusp

ABDF. (2020d). Recomendações técnicas sobre o covid-19 - sistema nacional de bibliotecas públicas. Recuperado de http://abdf.org.br/biblioteca-virtual-convide-i9/ vamos-aprender-algo/recomendacoes-tecnicas-sobre-o -covid-19-sistema-nacional-de-bibliotecas-publicas

ABDF. (2020e). Abnt libera lista de 32 normas gratuitas para contribuir no combate ao covid-19. Recuperado de http://abdf.org.br/biblioteca-virtual-convide-i9/ vamos-aprender-algo/abnt-libera-lista-de-32-normas -gratuitas-para-contribuir-no-combate-ao-covid-19

ABDF. (2020f). National library of medicine disponibiliza dados e notícias sobre o covid-19. Recuperado de http://abdf.org.br/biblioteca-virtual/biblioteca-virtual -convide-i9/vamos-aprender-algo/national-library-of -medicine-disponibiliza-dados-e-noticias-sobre-o-covid-19

ABDF. (2020g). Oxford university press - covid-19. Recuperado de http://abdf.org.br/biblioteca-virtual/ biblioteca-virtual-convide-i9/vamos-aprender-algo/ oxford-university-press-covid-19

Cunha, M. B., \& Cavalcanti, C. R. O. (2008). Dicionário de biblioteconomia e arquivologia. Brasília: Briquet de Lemos.

Delmazo, C., \& Valente, J. C. (2018). Fake news nas redes sociais online: propagação e reações à desinformação em busca de cliques. Media \& Jornalismo, 18(32), 155169. Recuperado de http://www.scielo.mec.pt/scielo.php ?script=sci_arttext\&pid=S2183-54622018000100012

National Library of Medicine. (2020). Accelerating biomedical discovery and data-powered health. Recuperado de https://www.nlm.nih.gov/

Organização Pan-Americana da Saúde. (2020). Entenda a infodemia e a desinformação na luta contra a covid-19. Recuperado de https://iris.paho.org/bitstream/handle/10665.2/ $52054 /$ Factsheet-Infodemic_por.pdf?sequence=14

Werthein, J. (2000). A sociedade da informação e seus desafios. Ciência da informação, 29(2), 71-77. Recuperado de https://www.scielo.br/pdf/ci/v29n2/a09v29n2.pdf

França, F. P., Araújo, D. O. \& Silva, M. C. (2020). A iniciativa digital CONVIDE-i9 no combate à infodemia de COVID-19: breves apontamentos de atuação. AtoZ: novas práticas em informação e conhecimento, 9(2), 248 - 252. Recuperado de: http://dx.doi.org/10.5380/ atoz.v9i2.76472 